\title{
The role of hardness and roughness on the wear of different CoCrMo counterfaces on UHMWPE for artificial joints
}

\author{
Victor A. González-Mora ${ }^{1}$, Michael Hoffmann ${ }^{1}$, Rien Stroosnijder ${ }^{1}$, F. Javier Gil ${ }^{2}$ \\ ${ }^{1}$ Institute for Health and Consumer Protection, Joint Research Centre, European Commision, Ispra, Italy; \\ ${ }^{2}$ Department of Materials Science and Matallurgical, Technical University of Catalonia, Barcelona, Spain. \\ Email: francesc.xavier.gil@upc.edu
}

Received 19 April 2011; revised 12 May 2011; accepted 2 September 2011.

\begin{abstract}
Wear tests were carried out to study the effect of the hardness and roughness with various counterface materials on UHMWPE wear behaviour. The materials used as counterfaces were based on varieties of CoCrMo: 1) forged (hand-polished) CoCrMo, 2) forged (mass-finished) CoCrMo, and 3) cast (massfinished) CoCrMo. Additionally, two coatings were proposed: 1) a CoCrMo coating applied to the forged CoCrMo alloy by means of physical vapour deposition (PVD), and 2) a $\mathrm{ZrO}_{2}$ coating applied to the forged CoCrMo alloy by means of plasma-assisted chemical vapour deposition (PACVD). The reciprocating pin-on-flat (RPOF) device for pin-on-disk wear testing was used for this study. The worn surfaces were observed using optical, atomic force and scanning electron microscopes.
\end{abstract}

Keywords: Wear; Artificial Joints; CoCrMo; Hardness; Roughness

\section{INTRODUCTION}

The total replacement of damaged or diseased synovial joints represents the greatest advance in orthopaedic surgery the last century [1]. The ability to replace damaged joints with prosthetic implants has brought relief to millions of patients who would otherwise have been severely limited in their most basic activities and resigned to a life of pain $[1,2]$.

Actual material combinations are based on a polymer component for the acetabular cup in the hip joint or the tibial plateau in the knee joint, and a metallic or ceramic counterface for the femoral head in the hip joint or the femoral condyle in the knee joint. Specifically for the polymeric component, the Ultra High Molecular Weight Polyethylene (UHMWPE) has been universally adopted. At present, the couple or sliding pair composed by UHMWPE and a metallic counterface (currently a CoCr- based alloy) are the most widely applied. This material combination is referred to as polyethylene-on-metal artificial joint. Another possibility for the counterface material is use of a ceramic material, this is referred to as a polyethylene-on-ceramic (alumina and zirconia are the most relevant ceramic materials). In the last years a renewed interest for two different concepts has developed. These are the metal-on-metal and ceramic-on-ceramic artificial joints $[3,4]$.

When the natural joint has to be replaced with artificial materials, there is a change in the tribological situation due to the inability of the actual materials used to produce an artificial permanent lubricating film. Therefore, the materials used for articulating components in an artificial joint are always subject to wear. Furthermore, there is no ideal bearing material that currently fulfils all the requirements of arthroplasty design [5-7]. Importantly therefore wear has to be minimised to avoid possible aseptic loosening following osteolysis due to particleinitiated foreign body reaction $[8,9]$.

The articulating surfaces of a total joint replacement are recognised as major sources of wear debris generation. Accurate laboratory wear simulations are essential for evaluating candidate materials and designs, because it is neither practical nor justified to evaluate the numerous potential design alternatives through clinical trials. By means of laboratory wear tests, useful tribological information can be produced for clinical assessment of new designs, materials, surface treatments, coatings, etc.

\section{MATERIALS AND METHODS}

A reciprocating pin-on-flat (RPOF) device is a special pin-on-disk (POD) wear tester that was designed in accordance with the ASTM F732-82 standard. This standard is the first specific standard in the field of biotribology. It sets guidelines for a "laboratory method for evaluation of the friction and wear properties of combinations of materials that are being considered for use as 
the bearing surfaces of human total joint replacement prostheses" $[6,7,10]$. The standard is intended mainly for the evaluation of polymer material combinations.

The RPOF wear-test device is a tribosystem, in which an apparatus produces an oscillatory relative motion between the pins and plates. Normally, the pins are stationary while the plates have an oscillating motion. The motion is always in a horizontal plane and unidirectional (Figure 1). These "reciprocating" devices are so called because of the reciprocating oscillating movement of the plate with respect to the pin.

The tests on the RPOF wear test method were performed as follows. Disks were mounted on a linear bearing while the pins were fixed and pressed against the disks. The motion on the RPOF machine is unidirectional and reciprocating over a stroke length of $17 \mathrm{~mm}$. A load of $225 \mathrm{~N}(23 \mathrm{Kg})$ was positioned over the pins. It results in a contact pressure of $3.5 \mathrm{MPa}$ considering a pin contact area of $63.6 \mathrm{~mm}^{2}$. The frequency of the motion was $1 \mathrm{~Hz}$, what results in the completion of a cycle per second. A cycle is considered after completion of two stroke lengths, that is, go and back motion of the disks. The wear of the UHMWPE pins was determined by weight loss measurements every 250,000 cycles up to a total test length of 1 million cycles, which corresponds to 1 year's life of the prosthesis [11].

The test lubricant was replaced with fresh solution after every weighing stop and distilled water was added during the test for compensating water evaporation. As test lubricant, a solution consisting of bovine serum and distilled water was used, which had a total protein concentration of $30 \mathrm{mg} / \mathrm{ml}$ simulating the clinical situation

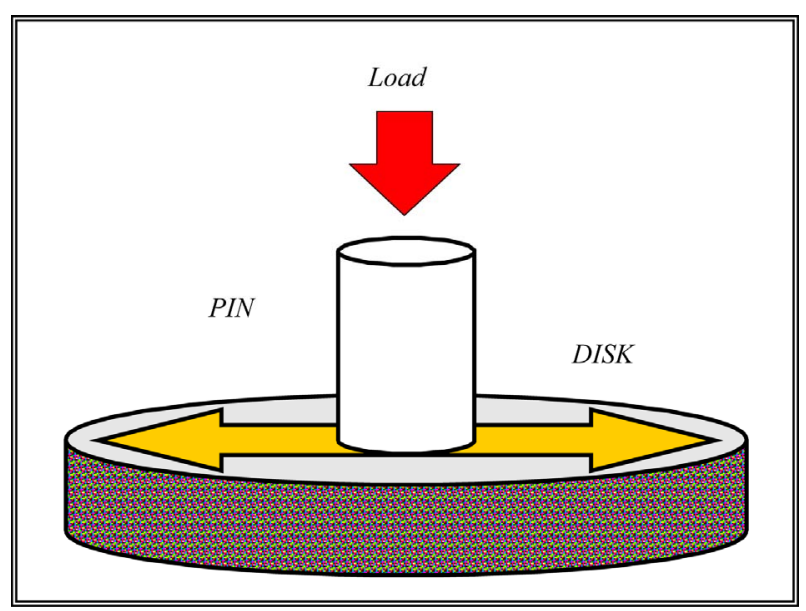

Figure 1. Motion/loading configuration of a RPOF wear test machine showing the translating unidirectional movement of the pin on the plate. The yellow arrow shows the direction of sliding and the red arrow shows the direction of the applied load.
[12]. The serum was purchased at Sigma-Aldrich SrI (Calf serum, bovine donor; product No.C9676). The soak adsorption of the UHMWPE pins was determined using an additional control pin, which was loaded identically as the UHMWPE pins in the RPOF machine, but no motion was applied. The cleaning and drying of the UHMWPE pins was performed according to the ASTM 1715 standard. Weighing was carried out with a Mettler Toledo AT261DeltaRange ${ }^{\circledR}$ microbalance with an accuracy of $10 \mu \mathrm{g}$.

Pins were manufactured from a medical grade UHMWPE GUR1120 bar, previously sterilized with standard, $25 \mathrm{KGy}$ (2.5 Mrad), gamma radiation. The dimensions of the pins were $13 \mathrm{~mm}$ length and $9 \mathrm{~mm}$ diameter. Disks were manufactured of five different material counterfaces, all of them being CoCrMo alloy. Test conditions and materials are resumed in Table 1.

The standard material in this study was a hot forged CoCrMo alloy; the chemical composition is given in Table 2.

CoCrMo forged (hand polished)

CoCrMo forged (mass finished)

CoCrMo cast (mass finished).

Additionally to the materials mentioned above, two coatings were proposed. The first one is a CoCrMo coating applied on the forged CoCrMo alloy by Physical Vapour Deposition (PVD). The coating had the same chemical composition than the substrate. The rational for this kind of coating is related to the use of

Table 1. Test conditions of the RPOF wear tests.

\begin{tabular}{|c|c|}
\hline Test parameter & Value \\
\hline Type of motion & Unidirectional (reciprocating) \\
\hline Contact geometry & Flat-on-flat \\
\hline Frequency & $1 \mathrm{~Hz}$ \\
\hline Sliding distance/cycle & $17 \mathrm{~mm}$ \\
\hline Contact area & $63.6 \mathrm{~mm}^{2}$ \\
\hline Applied load & $23 \mathrm{Kg}(225 \mathrm{~N})$ \\
\hline Contact stresses & $3.54 \mathrm{MPa}$ \\
\hline Test length & 1 million cycles (at intervals of 250,000 ) \\
\hline Lubricant & $30 \mathrm{mg} / \mathrm{ml}$ initial protein content \\
\hline Temperature & Room \\
\hline UHMWPE component & GUR1120 \\
\hline $\begin{array}{l}\text { Counterface } \\
\text { component }\end{array}$ & $\begin{array}{c}\text { CoCrMo forged (hand polished) } \\
\text { CoCrMo forged (mass finished) } \\
\text { CoCrMo cast } \\
\text { CoCrMo forged with a CoCrMo coating } \\
\text { CoCrMo forged with a } \mathrm{ZrO}_{2} \text { coating }\end{array}$ \\
\hline
\end{tabular}


Table 2. Chemical composition of the forged CoCrMo alloy in $\%$.

\begin{tabular}{ccccccccc}
\hline Element & $\mathrm{Cr}$ & $\mathrm{Mo}$ & $\mathrm{Mn}$ & $\mathrm{Ni}$ & $\mathrm{Si}$ & $\mathrm{Fe}$ & $\mathrm{C}$ & $\mathrm{N}$ \\
\hline \multirow{2}{*}{ Balance } & $26-30$ & $5-7$ & $\max$ & $\max$ & $\max$ & $\max$ & $\max$ & $\max$ \\
& & & 1 & 1 & 1 & 0.7 & 0.35 & 0.25 \\
\hline
\end{tabular}

femoral components in Total Knee Replacements (TKRs)

The other coating applied on the forged CoCrMo alloy was a $\mathbf{Z r O}_{2}$ coating applied by plasma assisted chemical vapour deposition (PACVD). The rationale for testing this coating is the same as for the CoCrMo coating CoCrMo forged with a $\mathrm{ZrO}_{2}$ coating

For each counterface material, four disks were tested and at less, three of them were considered for evaluation. A total of 40 wear tests were performed. The roughness and the hardness of each material can be observed in Table 3.

\section{EXPERIMENTAL RESULTS AND DISCUSSION}

The wear results obtained with the RPOF test method for the UHMWPE specimens (pins) are shown in Figure 2, where the volumetric wear $\left(\mathrm{mm}^{3}\right)$ of the UHMWPE pins is represented as a function of test duration (in cycles) and the different counterfaces. The volumetric wear results are calculated form the average weight loss of three specimens per each material.

The results show that the CoCrMo coating causes the highest UHMWPE wear of all counterfaces tested. The CoCrMo coating wear rates in an order of magnitude higher than that produced by the mass finished (forged) alloy, which in this study causes the least UHMWPE wear. The $\mathrm{ZrO}_{2}$ coating and the hand polished (forged) CoCrMo alloy produce intermediate UHMWPE wear rates. The wear rates show a UHMWPE wear value for the $\mathrm{ZrO}_{2}$ coating about the half of the CoCrMo coating. Standard deviations vary between 0.01 and $0.05 \mathrm{mg}$, except for the CoCrMo coating. The implication of this

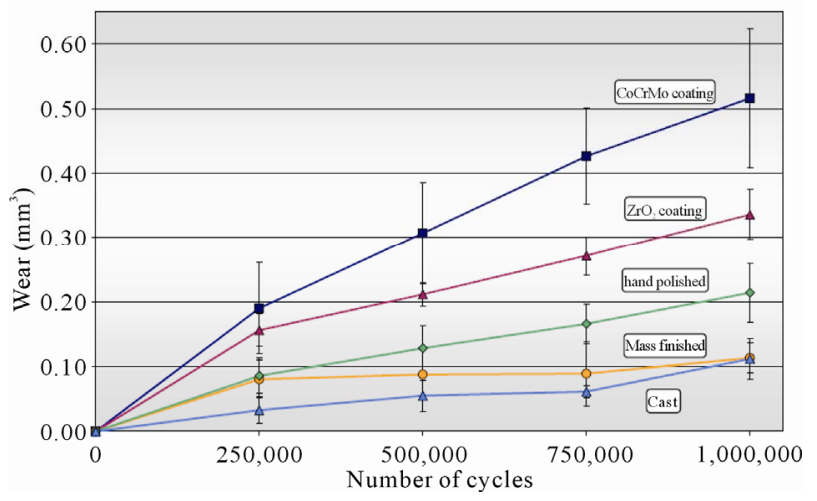

Figure 2. Average volumetric wear of UHMWPE pins sliding against different counterfaces. measurement variation is that with little weight loss of the UHMWPE the gravimetric wear determination is highly affected form the intrinsic uncertainty of the measurement. So that for the very early stages and especially for counterfaces producing very little weight loss of the UHMWPE specimen, the measurements are affected of a high uncertainty. Regarding the CoCrMo coating, it had significantly higher standard deviations ranging from 0.08 to $0.11 \mathrm{mg}$. The greater scatter for the CoCrMo coating is thought to be due to the higher sensitivity of the coating to scratches and third-body wear which highly influence the surface roughness of the disks and subsequently the weight loss of the UHMWPE.

The microhardness measurements were performed to investigate, whether a correlation between the UHMWPE wear and the Vickers microhardness of the counterface have been established. The results of Table 3 show that the mass finishing treatment on the surface of the forged CoCrMo alloy increases the microhardness of the material about $25 \%$ when compared with the hand polished forged, showing the increase in hardness by means of the mass finishing treatment. The reason of the hardness increase in mass finished alloys is due to the impact of the abrasive inert particles during the process, which produce a state of deformation on the surface. The CoCrMo coating shows the highest value while the $\mathrm{ZrO}_{2}$ coating shows the lower hardness value.

Hardness ranking agrees with the wear results and surface observations for the bulk counterfaces. From Figure 3 can be observed the relationship between the microhardness versus wear rates. Thus, the mass finished (forged) alloy causes less UHMWPE wear than the mass finished (cast) alloy and the later causes less UHMWPE wear, at least for the bulk material. In the same order, mass finished (forged) CoCrMo alloy is harder than the mass finished (cast) alloy and latter is harder than the hand polished (forged) alloy, as can be observed in Figure 3. As the figure shows, there is a linear ship between counterface hardness and UHMWPE wear, at least for the bulk materials. Therefore, the harder a surface is the less UHMWPE wear causes. The effect of the counterface hardness in the UHMWPE wear is due to the fact

Table 3. Roughness and hardness for each material tested.

\begin{tabular}{ccc}
\hline Material & Roughness Ra $(\mu \mathrm{m})$ & Hardness $(\mathrm{HVN})$ \\
\hline Hand Polished & $0.03 \pm 0.01$ & $673 \pm 21$ \\
Mass Polished & $0.05 \pm 0.01$ & $840 \pm 62$ \\
Cast CoCrMo & $0.05 \pm 0.01$ & $783 \pm 52$ \\
CoCrMo coating & $0.10 \pm 0.01$ & $884 \pm 28$ \\
$\mathrm{ZrO}_{2}$ coating & $0.06 \pm 0.01$ & $575 \pm 43$ \\
\hline
\end{tabular}




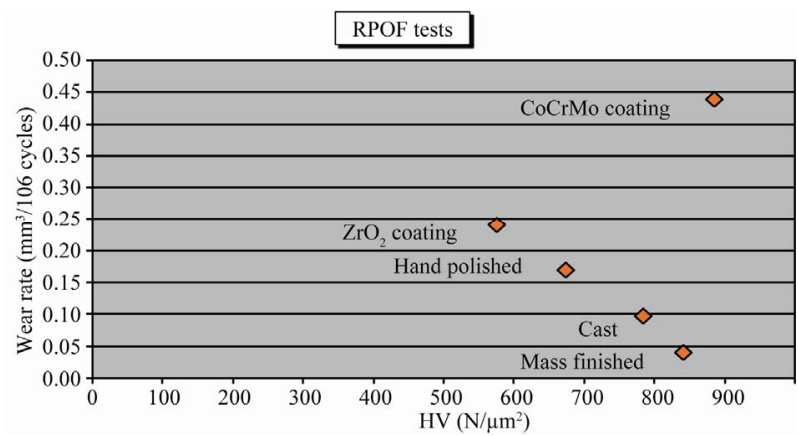

Figure 3. Vickers microhardness versus wear rate relationship in the RPOF wear test.

that hard surfaces are more resistant against scratching and consequently produces less UHMWPE wear, since an increase in the surface roughening produces an exponential increase in the UHMWPE wear. Indirectly, the results here discussed indicated that the main wear process occurring is abrasive wear and that adhesive wear is less important or sensitive.

From the material characterisation discussed before, it is clear that the hand polished counterface has a better surface finish than the mass finished counterfaces (both forged and cast). In a first instance, it is reasonable to think that a rougher surface of the mass finished counterface would produce a higher UHMWPE wear than the smother surface of the hand polished counterface, since the wear of an UHMWPE component depends on the material counterface's condition. However, the results of this study shown that this assumption is erroneous and that when predicting the effect in the UHMWPE wear of different counterface materials the counterface hardness are the essential parameter. On the contrary, the surface roughness of the counterfaces does not appear to be an important parameter when evaluating the UHMWPE wear produced by different counterfaces.

The influence of counterface hardness in the UHMWPE wear resulting from this study resembles the fact the ceramic counterface causes less UHMWPE wear than metallic counterfaces, even when having similar surface finishing. Evidence of reduced UHMWPE from ceramic counterfaces is given in the literature from both clinical and laboratory studies [1-4,13]. Hard, stable ceramic surfaces such as $\mathrm{Al}_{2} \mathrm{O}_{3}$ or $\mathrm{ZrO}_{2}$ can be expected to maintain their initial surface finish and thus minimise UHMWPE wear. On the other hand, metallic counterfaces can be scratched increasing thus the roughness of the counterface and the UHMWPE wear [12-15].

The UHMWPE wear caused by the $\mathrm{ZrO}_{2}$ coating agrees with the assumption that less harder counterfaces causes more UHMWPE wear. Additionally, AFM and SEM observation of the worn surfaces have shown superficial defects of the coating itself (Figure 4(a)) oc- curred during the coating deposition. These defects together with the irregular mass finished substrate surface and the well-known fragility of $\mathrm{ZrO}_{2}$ coatings can be the cause of the coating fracture and subsequent detachment, which can be observed in Figure 4(b). This kind of coating failure can produce a high UHMWPE wear. In Figure 5 can be observed by means AFM.

Regarding the CoCrMo coating, it has the highest hardness value. This should provide the coating a very good scratch resistance, at least much than for the bulk materials investigated. Under the prevailing experimental conditions, however, the CoCrMo coating produced the highest UHMWPE wear in this study. Additionally, the observation of the CoCrMo coating worn surface by AFM has shown a highly scratched CoCrMo coating surface and consequently an increase in surface rough-

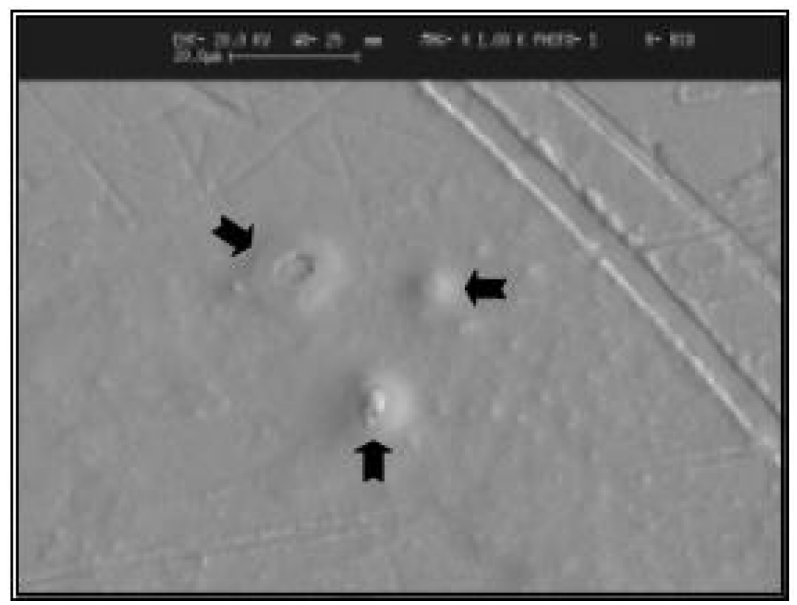

(a)

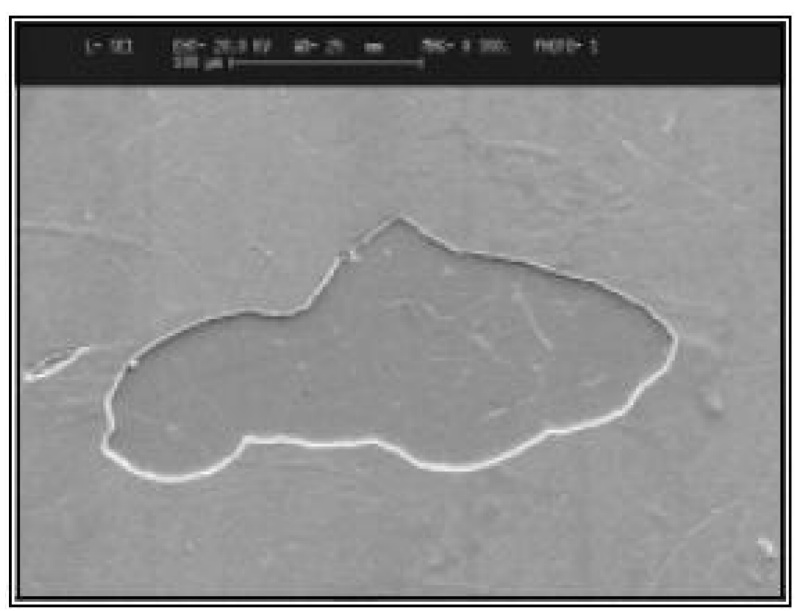

(b)

Figure 4. SEM micrographs of the $\mathrm{ZrO}_{2}$ coating surface. (a) As-received surface showing coating deposition defects see arrows. (b) Surface after the wear test showing the failure of the coating by fracture and detachment. The CoCrMo subtract was identified by EDS analysis. 


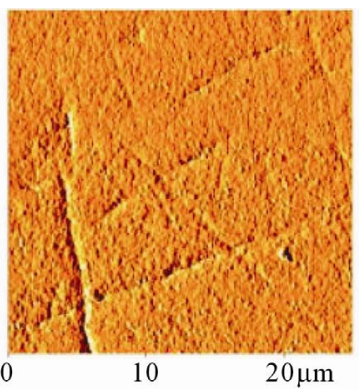

(a)

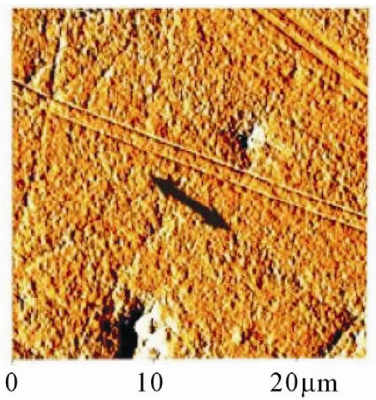

(c)

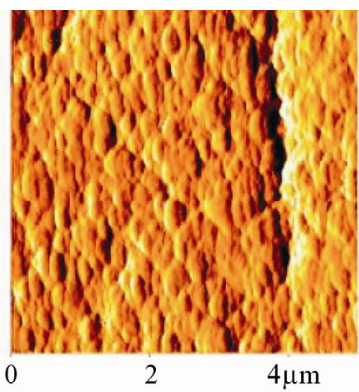

(b)

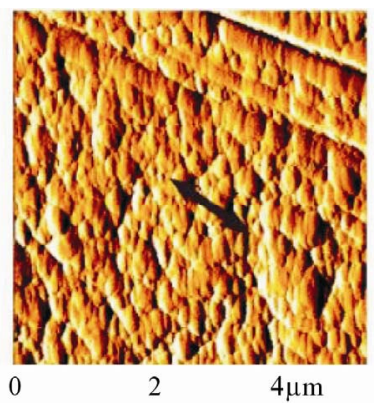

(d)

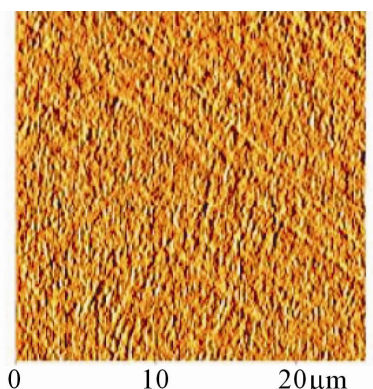

(a)

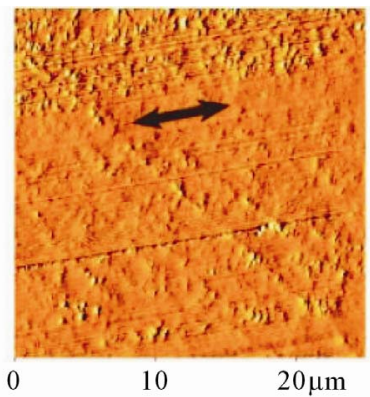

(c)

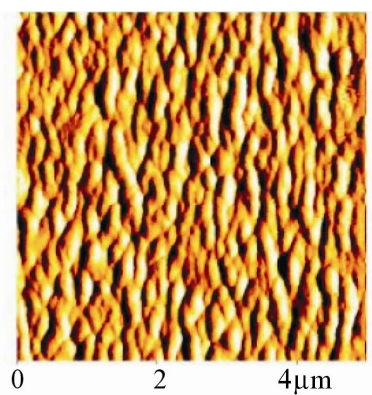

(b)

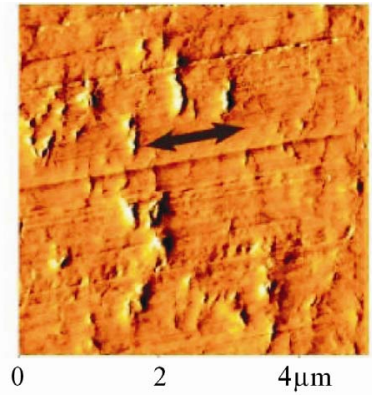

(d)

Figure 5. AFM images of the $\mathrm{ZrO}_{2}$ coating. (a) $25 \times 25 \mu \mathrm{m}$ image of the virgin surface. (b) $5 \times 5 \mu \mathrm{m}$ image of the virgin surface. (c) $25 \times 25 \mu \mathrm{m}$ image of the surface on the wear track. (d) $5 \times 5 \mu \mathrm{m}$ image of the surface on the wear track. The black arrows show the direction of sliding.

ness, causing high UHMWPE wear. Furthermore, coating fragments may have favour third body wear mechanisms, roughening too the coating surface.

The as-received surface of the CoCrMo coating appears very homogenous. The scratches left during the mass finishing process are not present, since the coating has covered them (Figures 6(a) and (b)), leaving the nodules as typical features of the coating deposition. Compared to the forged CoCrMo alloy, the surface of the CoCrMo PVD coating has undergone a significant change after the wear test (Figures 6(c) and 6(d)). The homogeneous structure of the CoCrMo coating in the as-received state has completely disappeared and scratches parallel to the sliding direction have formed. It has been supposed that these scratches have been likely produced by parts of the coating, which had delaminated from the coating surface, leading to third-body wear. This possibility corroborates the higher number of scratches seen by the optical microscope on this material.

Additionally, ridges perpendicular to the sliding direction have remained on the coating surface. These are the rests of the nodules left on the coating deposition. Both, the ridges and scratches are considered responsible for the observed increase in the surface roughness, causing the higher UHMWPE wear compared to the forged and cast CoCrMo alloy.

Figure 6. AFM images of the CoCrMo coating. (a) $25 \times 25 \mu \mathrm{m}$ image of the virgin surface. (b) $5 \times 5 \mu \mathrm{m}$ image of the virgin surface. (c) $25 \times 25 \mu \mathrm{m}$ image of the surface on the wear track. (d) $5 \times 5 \mu \mathrm{m}$ image of the surface on the wear track. The black arrows show the direction of sliding.

\section{CONCLUSIONS}

An indirect relationship between counterface hardness and UHMWPE wear has been found. The effect of the counterface hardness in the UHMWPE wear is due to the fact the hard surfaces are more resistant against scratching and consequently produces less UHMWPE wear. The results of this study have shown that the UHMWPE wear caused by different counterface materials is mainly determined by the counterface hardness. The roughness is not the main parameter.

\section{REFERENCES}

[1] Howie, D. and McGee, M. (1996) Wear and osteolysis in relation to prostheses design and materials. Medical Applications of Titanium and Its Alloys (ASTM STP 1272).

[2] McGee, M., Howie, D., Neale, S., Haynes, D. and Pearcy, M. (1997) The role of polyethylene wear in joint replacement failure. Proceedings of the Institution of $\mathrm{Me}$ chanical Engineering, Part H: Journal of Engineering in Medicine, 211, 65-72.

doi:/10.1243/0954411971534692

[3] Dowson, D. and Wallbrigde, N. (1985) Laboratory wear tests and clinical observations of the penetration of femoral heads into acetabular cups in total replacement hip joints: I: Charnley prostheses with polytetrafluoroethylene acetabular cups. Wear, 104, 203-215. doi:/10.1016/0043-1648(85)90048-1 
[4] Zhou, Y., Ikeuchi, K. and Ohashi, M. (1997) Comparison of the friction properties of four ceramic materials for joint replacements. Wear, 210, 171-177. doi:/10.1016/S0043-1648(97)00066-5

[5] Wang, A., Polineni, V., Essner, A., Sokol, M., Sun, D., Stark, C. and Dumbleton, J. (1997) The significance of nonlinear motion in the wear screening of prthopaedic implants materials. Testing \& Evaluation, 13, 239-245.

[6] Wang, A., Stark, C. and Dumbleton, J. (1996) Mechanistic and morphological origins of ultra-high molecular weight polyethylene wear debris in total joint replacement. Proceedings of the Institution of Mechanical Engineerng, Part H: Journal of Engineering in Medicine, 210, 141-156.

[7] Murray, D., Rushton, N. (1990) Macrophages stimulate bone resorption when they phagocytose particles. Journal of Bone and Joint Surgery, 72, 988-992.

[8] Green, T., Fisher, J., Stone, M., Wroblewski, B. and Ingham, E. (2004) Polyethylene particles of a "critical size" are necessary for the induction of cytokines by macrophages in vitro. Biomaterials, 19, 2297-2302. doi:/10.1016/S0142-9612(98)00140-9

[9] Doorn, P., Mirra, J., Campbell, P. and Amstutz, H. (1996) Tissue reaction to metal on metal total hip prostheses. Clinical Orthopaedics Related Research, 329, 187-205. doi:/10.1097/00003086-199608001-00017

[10] Gil, F.J., Manero, J.M. and Planell, J.A. (1995) Tissue-
Effect of grain size on the martensitic transformation in NiTi alloy. Journal of Materials Science, 30, 2526- 2530. doi:/10.1007/BF00362129

[11] Gil, F.J. and Planell, J.A. (1999) Effect of copper addition on the superelastic behavior of $\mathrm{Ni}$ - Ti shape memory alloys for orthodontic applications. Journal of Biomedical Materials Resesrch, 48, 682-688.

doi:/10.1002/(SICI)1097-4636(1999)48:5<682::AID-JB $\mathrm{M} 12>3.0 . \mathrm{CO} ; 2-\mathrm{M}$

[12] Viceconti, M. Cavallotti, G., Andrisano, A. and Toni, A. (1996) Discussion on the design of a hip joint simulator. Medical Engineering and Physics, 18, 234-240. doi:/10.1016/1350-4533(95)00026-7

[13] Wang, A., Essner, A., Polineni, V., Stark, C. and Dumbleton, J. (1998) Lubrication and wear of ultra-high molecular weight polyethylene in total joint replacements. Tribology International, 31, 17-33. doi:/10.1016/S0301-679X(98)00005-X

[14] Gil, F.J. and Planell, J.A. (1998) Shape memory alloys for medical applications. Proceedings of the Institution of Mechanical Engineering, Part H: Journal of Engineering in Medicine, 212, 473-488. doi:/10.1243/0954411981534231

[15] Guilemany, J.M. and Gil, F.J. (1991) Kinetic grain growth in $\mathrm{Cu}-\mathrm{Zn}$-Al shape memory alloys. Journal of Materials Science, 26, 4626-4630. doi:/10.1007/BF00612397 